\title{
PROTECTION PAR VOIE ÉLECTROCHIMIQUE DE HUBLOTS IMMERGÉS EN MILIEU MARIN VIS-À-VIS DES BIOSALISSURES
}

\author{
H. Cachet ${ }^{1}$, D. Festy ${ }^{2}$, G. Folcher' ${ }^{\prime}$ F. Mazeas $^{2}$ et B. Tribollet' \\ 1. CNRS-UPR 15, Physique des Liquides et Electrochimie, Université P. el M. Curie, Paris \\ 2. IFREMER, Centre de Brest, Service Matériaux et structures, Plouzané
}

\section{ELECTROCHEMICAL PROTECTION \\ OF WINDOWS IMMERSED IN SEAWATER AGAINST BIOFOULING}

ABSTRACT: Biofouling reduction is a major problem for underwater instrumentation, involving optical measurements or viening. This paper presents an effective window protection technique based on seawater electrolysis for producing active chlorine species. This is achioled by depositing a transparen conductive in dioxide coating by spray pyrolysis. A number of laboratory and in situ experiments were performed in order to assess the effectiveness of this window protection technique. It was found that Sb doping was more efficient with respect to electrical energy management and long term $\mathrm{SnO}_{2}$ coating stability than the fluorine doping. In the present state of the art, biofouling prevention was proved to be effective over 5 momths al least.

\begin{abstract}
RÉSUMÉ: La prévention du biofilm est un problème majeur dans la mise en ceuvre d'équipements sous-marins impliquant des mesures optiques ou simplement l'obtention d'images. Le présent article a pour but de décrire brièvement les travatux qui ont permis de valider une technique de protection par voie électrochimique. Le principe repose sur une électrolyse contrólée de l'eau de mer pour produire des espèces chlorées à la surface même de la fenêtre à protéger. Ceci est réalisé en déposant par la technique de pyrolyse de spray un film de dioxyde d'étain. transparent et conducteur. Sur la base de nombreuses études en laboratoire et sur des sites naturels, la validité du procédé a été démontrée. En particulier, il a été élabli que le dopage à l'antimoine offrait la meilleure stabilité et permettait de minimiser la consommation en énergie électrique. Dans l'état de l'art actuel, on a pu maintenir la transparence de fenêtres immergées en milieu marin pendant 5 mois au moins.
\end{abstract}

\section{Position du problème}

Conférence présentée au Forum "Biodétérioration des matériaux" organisé les 23 et 24 mai 2002 à Dourdan par la Commission Biodétérioration des Matériaux du Centre français de l'Anticorrosion (CEFraCOR) avec le parrainage de la Société Française de Microbiologie.
$\mathrm{L}$ a formation d'un biofilm réduit la durée de vie opérationnelle des éléments optiques immergés en milieu marin. La figure 1 montre l'état d'un hublot en verre laissé à l'abandon par 10 mètres de fond en rade de Brest pendant 1 mois. La surface est complètement recouverte d'un biofilm totalement opaque. Qu'il s'agisse de caméras de surveillance ou de capteurs optiques, il est donc de première importance de pouvoir empêcher la présence de biosalissures et de conserver toute la transparence de la fenêtre pendant plusieurs mois.

Face à la technique du nettoyage mécanique nécessitant une intervention humaine. ou à la dispersion en volume d'un produit biocide au voisinage du hublot à protéger et perturbant l'environnement, on a cherché à développer un procédé ne présentant pas ces deux inconvénients majeurs. Il repose sur l'idée simple qui consiste à oxyder électrochimiquement les ions chlorure présents dans l'eau de mer pour produire du chlore 


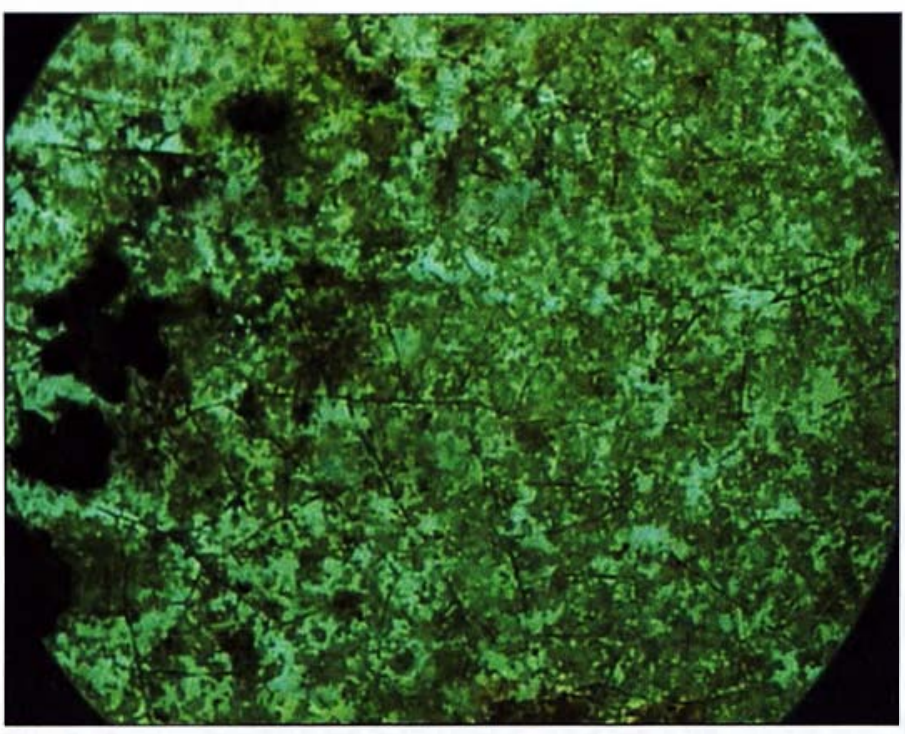

Fig. 1 - Recouvrement par le biofilm d'un hublot de verre initialement transparent après une immersion d'un mois par dix mètres de fond en rade de Brest.

Fig. I - Fouled surface of a glass window afier I month seawater exposure at 10 meter depth in the Brest bay.

actif, sous forme d'acide hypochloreux, à la surface même de la fenêtre à protéger.

L'électrolyse de l'eau de mer produit de l'oxygène $\left(\mathrm{O}_{2}\right)$, de l'acide hypochloreux $(\mathrm{HClO})$, des ions hypochloreux $\left(\mathrm{ClO}^{-}\right)$et des protons $\left(\mathrm{H}^{+}\right)$suivant les réactions :

$$
\begin{gathered}
2 \mathrm{H}_{2} \mathrm{O} \rightarrow \mathrm{O}_{2}+4 \mathrm{H}^{+}+4 \mathrm{e}^{-} \\
\mathrm{Cl}^{-}+\mathrm{H}_{2} \mathrm{O} \rightarrow \mathrm{HClO}+\mathrm{H}^{+}+2 \mathrm{e}^{-} \\
\mathrm{Cl}^{-}+\mathrm{H}_{2} \mathrm{O} \rightarrow \mathrm{ClO}^{-}+2 \mathrm{H}^{+}+2 \mathrm{e}^{-}
\end{gathered}
$$

D'un point de vue thermodynamique, la réaction (3) est prédominante aux potentiels supérieurs à $1 \mathrm{~V} / \mathrm{ECS}$ pour un $\mathrm{pH}$ voisin de 8 [1]. Du fait de la production d'ions $\mathrm{H}^{+}$, le $\mathrm{pH}$ peut localement décroître et favoriser la réaction (2), le pKa de l'acide hypochloreux étant 7,4 .

Par ailleurs, l'acide hypochloreux $\mathrm{HClO}$ peut réagir avec les ions bromure présents dans l'eau de mer pour former de l'acide hypobromeux, qui est aussi un produit biocide.

La mise en cuvre du procédé électrochimique nécessite de rendre conductrice la surface du hublot en verre au contact de l'eau, tout en maintenant la transparence du dispositif dans le visible $[2,3]$. La solution proposée consiste à déposer une couche mince d'un matériau à large bande interdite, que l'on puisse doper pour le rendre suffisamment conducteur et l'utiliser comme anode. La conductivité doit être suffisante pour que la couche servant d'électrode soit effectivement équipotentielle, c'est-à-dire que les effets de chute ohmique dans la couche soient négligeables compte tenu des dimensions géométriques et des densités de courant requises. Les oxydes conducteurs à grands gaps sont a priori de bons candidats pour l'application envisagée. Toutefois, parmi tous les oxydes couramment utilisés comme électrode transparente dans des dispositifs optoélectroniques ou photovoltaïques (oxydes de zinc, d'indium, d'étain), seul le dioxyde d'étain $\mathrm{SnO}_{2}$ s'est avéré correspondre au cahier des charges posé par le procédé de chloruration électrochimique. Le présent article décrit l'ensemble des travaux effectués dans le cadre d'une collaboration IFREMER-CNRS, qui ont conduit à la mise en œuvre opérationnelle de hublots en verre protégés électrochimiquement.

\section{Le film de dioxyde d'étain : élaboration et dopage}

Les films de dioxyde d'étain sont élaborés par la méthode d: pyrolyse d'un aérosol contenant le précurseur étain $\left(\mathrm{SnCl}_{4}\right)$ sur un substrat porté à une température comprise entre $400 \mathrm{El}$ $600^{\circ} \mathrm{C}$.

L'aérosol est formé à partir d'une solution de tétrachlorur: d'étain dans le méthanol par pulvérisation à l'aide d'un dispo sitif pneumatique utilisant l'azote comme gaz vecteur. La su face utile pour l'obtention de couches uniformes est d'enviro । $7 \times 7 \mathrm{~cm}^{2}$. La vitesse de croissance des couches est de l'ordr de $1 \mathrm{~nm} . \mathrm{s}^{-1}$

Le dopage intentionnel est réalisé soit par ajout de $\mathrm{NH}_{4}$ (dopage par le fluor en substitution de l'oxygène dans le résea $\mathrm{SnO}_{2}$ ), soit par ajout de $\mathrm{SbCl}_{3}$ (dopage par l'antimoine, en sut stitution de l'étain). Le dopage fluor $\left(\mathrm{SnO}_{2}: \mathrm{F}\right)$ permet d'atteindr les plus faibles valeurs de résistivité $\left(4\right.$ à $\left.6.10^{-4} \Omega . \mathrm{cm}\right)$ dans le , conditions suivantes: rapport atomique $\mathrm{F} / \mathrm{Sn}=0,7$ en solutio pour un taux d'incorporation effectif $1<\mathrm{F} / \mathrm{Sn}<2 \%$, une tem pérature de substrat de $480^{\circ} \mathrm{C}$. L'antimoine peut être incorpor en grande quantité (rapport atomique $0<\mathrm{Sb} / \mathrm{Sn}<30 \%$ ) dan la matrice de l'oxyde d'étain, au prix cependant d'une densit croissante de défauts structuraux limitant fortement la mobilit des porteurs. Avec le dopage $\mathrm{Sb}\left(\mathrm{SnO}_{2}: \mathrm{Sb}\right)$, l'optimum d

Tableau I - Propriétés électriques des films $\mathrm{SnO}_{2}$ déposés par spray । dopés au fluor ou à l'antimoine [2].

Table I - Electrical properties of $\mathrm{SnO}_{2}$ films spray-deposited and fluorineantimony-doped [2].

\begin{tabular}{|c|c|c|c|c|c|c}
\hline & $\mathbf{S n O}_{2}: \mathbf{F}$ & \multicolumn{5}{|c}{$\mathrm{SnO}_{2}: \mathbf{S b}$} \\
\hline $\begin{array}{c}\text { Taux de } \\
\text { dopage en \% }\end{array}$ & 70 & 1 & 2 & 3 & 4 & 5 \\
\hline $\begin{array}{c}N \\
\times 10^{-20} \mathrm{~cm}^{-3}\end{array}$ & 4,0 & 2,7 & 7,5 & 13,5 & 19 & 21 \\
\hline $\begin{array}{c}\text { Mobilité } \\
\mathrm{cm}^{2} \mathrm{~V}^{-1} \mathrm{~s}^{-1}\end{array}$ & 30 & 19 & 8 & 4 & 3 & 2,5 \\
\hline $\begin{array}{c}\text { Conductivité } \\
\Omega^{-1} \mathrm{~cm}^{-1}\end{array}$ & 1920 & 800 & 950 & 865 & 850 & 840 \\
\hline
\end{tabular}

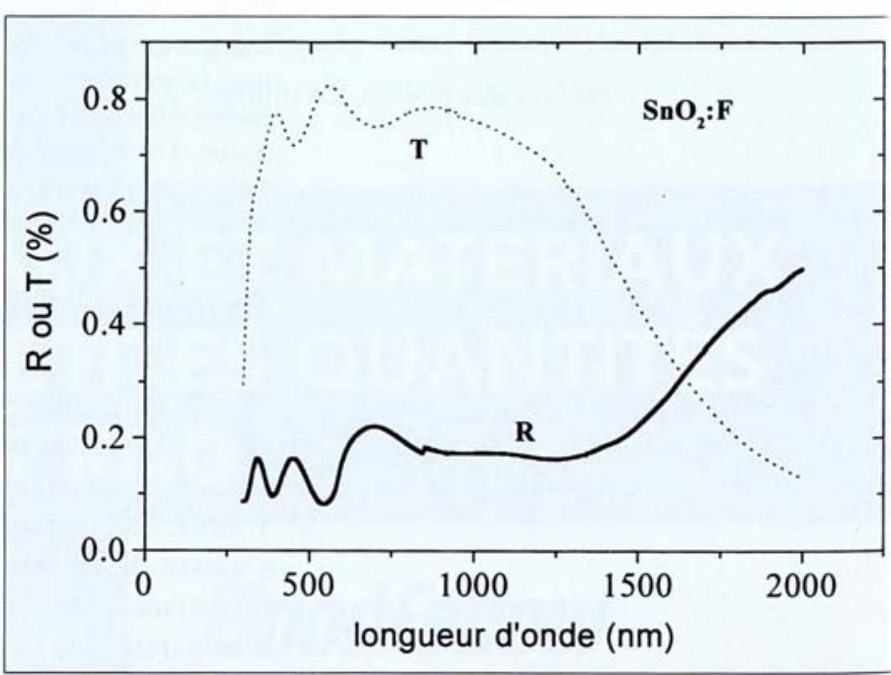

Fig. 2 - Courbes de transmission et de réflexion d'une couche $\mathrm{SnO}_{2}$ d'épaisseur $300 \mathrm{~nm}$, déposée sur verre et dopée par le fluor. La longueu d'onde plasma correspond à l'intersection des deux courbes.

Fig. 2 - Transmission and reflexion cunes of a $300 \mathrm{~nm}$ thick, fluorine dopen $\mathrm{SnO}_{2}$ layer deposited on a $2 \mathrm{~mm}$ thick glass slide. Plasma wavelength is given by the intersection of the two cumes. 
résistivité ( 8 à $\left.10.10^{-4} \Omega . c m\right)$, tout en gardant une bonne transparence, correspond à un rapport $\mathrm{Sb} / \mathrm{Sn}$ de 1 à $2 \%$, pour une température de substrat dans la gamme $500-570^{\circ} \mathrm{C}[4,5]$. Le tableau I regroupe des valeurs typiques de la concentration en électrons de conduction $\mathrm{N}$, de leur mobilité et de la conductivité des films $\mathrm{SnO}_{2}$ en fonction du type et de la concentration de dopant. La figure 2 donne les variations de la transmission et de la réflexion d'un film $\mathrm{SnO}_{2}$ dopé au fluor, d'épaisseur $300 \mathrm{~nm}$. La chute de la transmission aux longueurs d'onde supérieures à $1 \mu \mathrm{m}$ est due à l'absorption par les électrons de conduction; elle est caractéristique des couches fortement dopées, comme celles considérées dans le présent travail. L'intersection des zourbes de transmission et de réflexion définit la fréquence plasma, qui caractérise la limite au-delà de laquelle les électrons ne sont plus capables d'écranter le champ électrique associé à la radiation électromagnétique incidente. Elle est fonction de la densité de porteurs et de leur masse effective. Si cette dernière est connue, le simple tracé des courbes de réflexion-transmission permet d'obtenir la fréquence plasma et donc de connaître la concentration en électrons de conduction.

\section{Comportement anodique des films $\mathrm{SnO}_{2}$ et stabilité}

La figure 3 montre la réponse électrochimique de films $\mathrm{SnO}_{2}$ dopés au fluor et à l'antimoine (à $\mathrm{Sb} / \mathrm{Sn}=1 \%$ ) sous polarisation anodique, au contact d'eau de mer naturelle filtrée. Pour mémoire, le potentiel d'oxydation des ions chlorure s'établit à $+1,10$ V/ECS [6]. Les films dopés Sb sont beaucoup plus actifs électrochimiquement que ceux dopés au fluor. Dans le cas du dopage $\mathrm{Sb}$, la vague d'oxydation des chlorures se situe vers $+1,4 \mathrm{~V} / \mathrm{ECS}$, en se superposant au dégagement d'oxygène. Dans le cas du fluor par contre, cette même vague n'apparaît qu'à des potentiels d'au moins $2,5 \mathrm{~V} / \mathrm{ECS}$. La différence de réactivité peut s'expliquer par la présence d'une forte densité d'états électroniques présents dans la bande interdite de $\mathrm{SnO}_{2}$

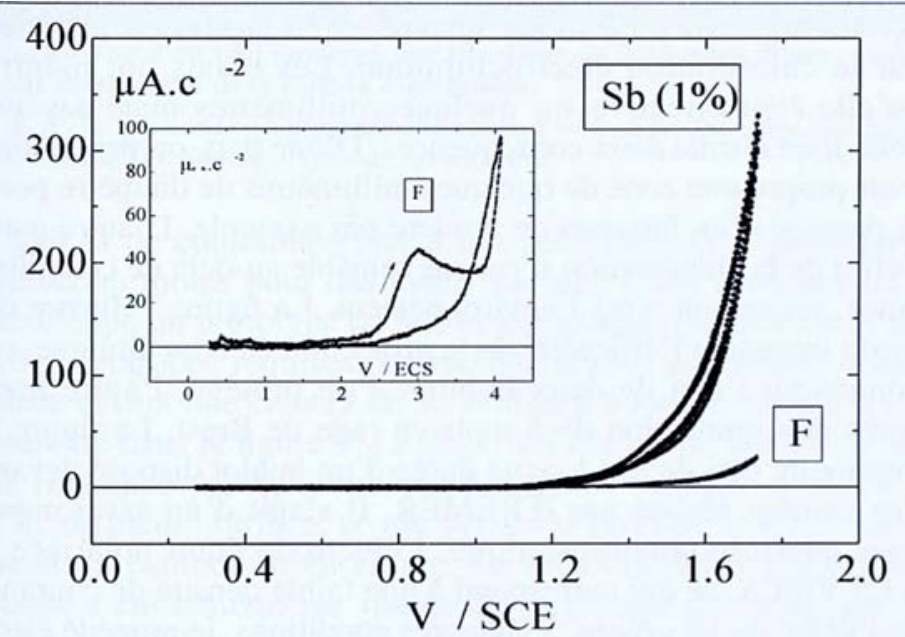

Fig. 3 - Réponse électrochimique de couches $\mathrm{SnO}_{2}$ respectivement dopées au fluor $(\mathrm{F})$ et à l'antimoine $(\mathrm{Sb} / \mathrm{Sn}=1 \%)$ au contact d'eau de mer naturelle filtrée. Vitesse de balayage du potentiel : $1 \mathrm{mV} . \mathrm{s}^{-1}$. L'encadré montre la vague d'oxydation des jons chlorure au-dela de 2,5 V/ECS dans le cas de l'électrode $\mathrm{SnO}_{2}$ dopée fluor.

Fig. 3 - Electrochemical behaviour of fluorine-doped and antimony-doped $(\mathrm{Sb} / \mathrm{Sn}=1 \%) \mathrm{SnO}_{2}$ films in natural filtered seawater. Potential scan rate $1 \mathrm{mVs} \mathrm{s}^{-1}$. The insert shows the chloride oxidation wave to be located at potentials more positive than 2,5 V/SCE for fluorine-doped $\mathrm{SnO}_{2}$. créés par les atomes d'antimoine incorporés. L'usage du dopage $\mathrm{Sb}$ apporte d'emblée un gain d'au moins I Volt par rapport au dopage fluor vis-à-vis de la réaction d'oxydation des ions chlorure.

Un autre aspect très important concerne la stabilité des couches $\mathrm{SnO}_{2}$ lorsqu'elles sont soumises à des polarisations anodiques. Pour cela, une étude in situ de la corrosion de $\mathrm{SnO}_{2}$ en milieu chloruré a été entreprise à l'aide d'une microbalance à quartz. Des électrodes $\mathrm{SnO}_{2}$ ont été directement déposées sur des quartz piézoélectriques, avec ou sans sous-couche métallique d'or ou de titane $[7,8]$. La variation de masse de l'électrode de travail a été enregistrée en fonction du temps, en cyclant le potentiel par balayage linéaire à des vitesses de 1 à $20 \mathrm{mV} \cdot \mathrm{s}^{-1}$. Dans ces conditions, le potentiel étant une fonction linéaire du temps, la vitesse de corrosion s'obtient par différentiation numérique des courbes masse-potentiel. La méthode de dérivation introduit certes du bruit mais permet de faire ressortir un comportement général illustré dans la figure 4. Cette figure, obtenue en milieu $\left[\mathrm{Cl}^{-}\right]=0,1 \mathrm{M}$, montre le profile très particulier de la vitesse de corrosion qui présente un pic principal centré vers $+1,2 \mathrm{~V} / \mathrm{ECS}$. Un second pic, d'amplitude beaucoup plus faible, apparaît vers $+1,4 \mathrm{~V} / \mathrm{ECS}$. Remarquablement, la vitesse de corrosion est quasiment nulle en dehors de ces deux pics. Du point de vue application, on choisira un mode de fonctionnement potentiostatique en polarisant à un potentiel en dehors de la région où la corrosion est la plus active. L'influence de la concentration en ions chlorure est illustrée par la figure 5 . On constate avec intérêt que la vitesse maximale de corrosion, prise au sommet du pic le plus élevé, décroît notablement lorsque la concentration en chlorure est supérieure $\grave{a} \approx 0,3 \mathrm{M}$. On note également que la stabilité est améliorée si l'on augmente la teneur en antimoine, sachant qu' au-delà d'une teneur $\mathrm{Sb} / \mathrm{Sn}=4 \%$, les films perdent à la fois en conductivité et en transparence. D'après la figure 5, on constate que la vitesse maximale de corrosion en milieu marin, mesurée à $+1,2 \mathrm{~V} / \mathrm{ECS}$, s'établirait à environ $1 \mu \mathrm{m} / \mathrm{an}$, ce qui est de l'ordre de grandeur de l'épaisseur des couches déposées. En polarisant à un potentiel de 1,5 V/ECS, la vitesse de corrosion est au moins 10 fois inférieure à cette valeur, garantissant ainsi une durée de vie opérationnelle d'au moins une année pour des films d'environ

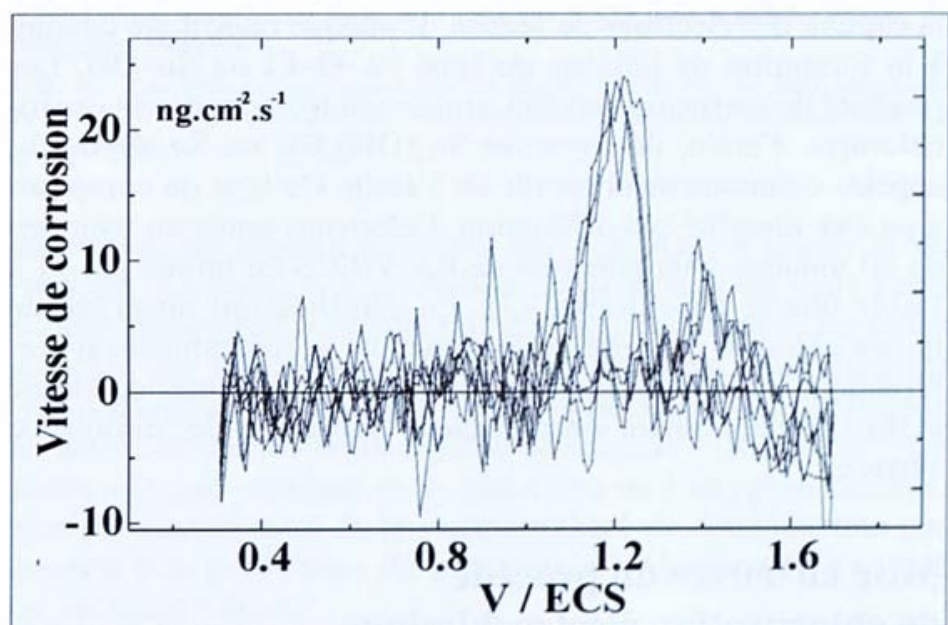

Fig. 4 - Profil de la vitesse de corrosion avec le potentiel déterminé par électrogravimétrie pour un film $\mathrm{SnO}_{2}: \mathrm{Sb}(1 \%)$ au contact d'une solution $0,1 \mathrm{M} \mathrm{LiCl}+0,9 \mathrm{M} \mathrm{LiClO}_{4}$ à $\mathrm{pH} 8$.

Fig. 4 - Potential dependence of the corrosion rate of a $\mathrm{SnO}_{2}: \mathrm{Sb}(1 \%)$ film in contact with a $0.1 \mathrm{M} \mathrm{LiCl}+0.9 \mathrm{M} \mathrm{LiClO}_{4} \mathrm{pH}=8$ solution, as determined by an in situ electrochemical quartz crnstal microbalance. 


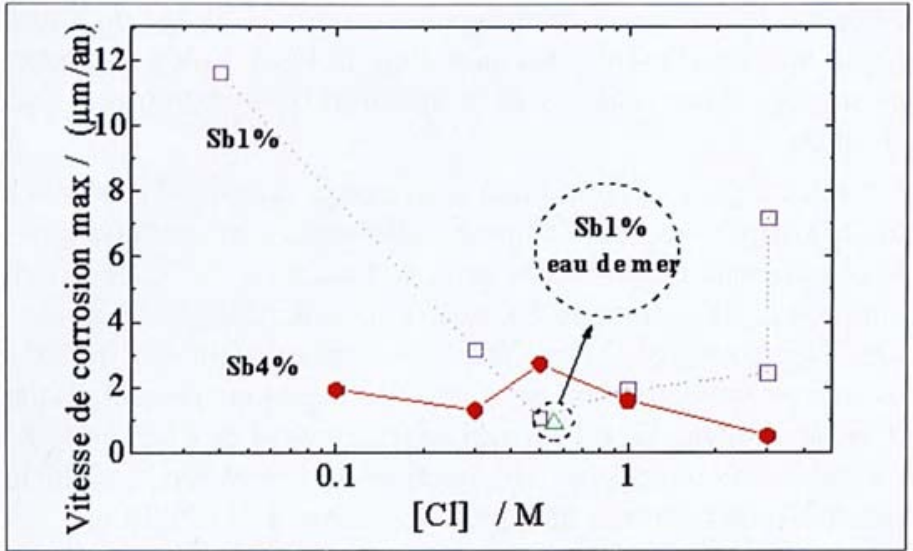

Fig. 5 - Variations de la vitesse maximale de corrosion, mesurée au sommet du pic illustré dans la figure 4 , en fonction de la concentration en ions chlorure.

Fig. 5 - Variations of the peak corrosion rate (as depicted in fig.4) with the chloride concentration.

$400 \mathrm{~nm}$ d'épaisseur. Ces estimations ont été confirmées par les essais sur site menés par IFREMER.

Le mécanisme de corrosion du dioxyde d'étain en milieu aqueux chloruré à un $\mathrm{pH}$ de 8 et soumis à des potentiels plus positifs que $0,9 \mathrm{~V} / \mathrm{ECS}$ a fait l'objet d'une étude approfondie pour comprendre l'origine de l'effet stabilisateur observé aux fortes concentrations en chlorure [8]. Le mécanisme qui a été proposé repose sur l'existence de deux chemins réactionnels symétriques se déroulant en parallèle. L'un concerne la transformation hydroxyde/oxygène et l'autre la transformation chlorure/chlore. Pour chacun de ces deux chemins, on a considéré quatre étapes successives. La première étape rend compte de la formation d'un radical $A^{\circ}$ par oxydation électrochimique d'un anion $\mathrm{A}^{-}\left(\mathrm{A}^{-}=\mathrm{OH}^{-}\right.$ou $\left.\mathrm{Cl}^{-}\right)$. La seconde étape traduit l'adsorption chimique de ce radical sur un site de surface $X$ en formant un groupement $\mathrm{X}-\mathrm{A}^{\circ}$. La troisième étape réside dans la séparation des charges dans la liaison $\mathrm{X}-\mathrm{A}^{\circ}$ en créant un site de surface $\mathrm{X}^{\oplus}$ chargé positivement et en libérant un anion $\mathrm{A}^{-}$. La dernière étape porte sur la régénération du site de surface $\mathrm{X}$ accompagnée d'un dégagement gazeux $\left(\mathrm{O}_{2}\right.$ ou $\left.\mathrm{Cl}_{2}\right)$. La corrosion de $\mathrm{SnO}_{2}$ est attribuée à l'action combinée de radicaux $\mathrm{OH}^{\circ}$ et $\mathrm{Cl}^{\circ}$ sur les liaisons de surface $\mathrm{Sn}-\mathrm{O}$ de l'oxyde, affaiblies par la capture des électrons de liaison. L'attaque radicalaire conduit à la formation de liaisons du type $\mathrm{Sn}-\mathrm{O}-\mathrm{Cl}$ ou $\mathrm{Sn}-\mathrm{OH}$. Les produits de corrosion seraient vraisemblablement des hydroxochlorures d'étain, de formules $\mathrm{Sn}_{4}(\mathrm{OH})_{6} \mathrm{Cl}_{2}$ ou $\mathrm{Sn}_{3}(\mathrm{OH})_{2} \mathrm{Cl}_{2}$, appelés communément rouille de l'étain. Ce type de composés a pu être identifié par diffraction d'électrons après un maintien de 20 minutes à un potentiel de 0,8 V/ECS en milieu pH 11 à faible teneur en chlorure [7]. La stabilisation observée en milieu chlorure concentré s'explique par la recombinaison préférentielle entre radicaux conduisant à la production de chlore et d'oxygène, limitant ainsi l'attaque radicalaire des liaisons de surface.

\section{Mise en ouvre du procédé de chloruration électrochimique}

Afin d'optimiser à la fois la conduction et la stabilité à long terme de la couche $\mathrm{SnO}_{2}$ déposée en face avant d'un hublot en verre, on a mis au point une procédure de dépôt par spray en deux étapes. La première étape consiste en un dépôt d'une

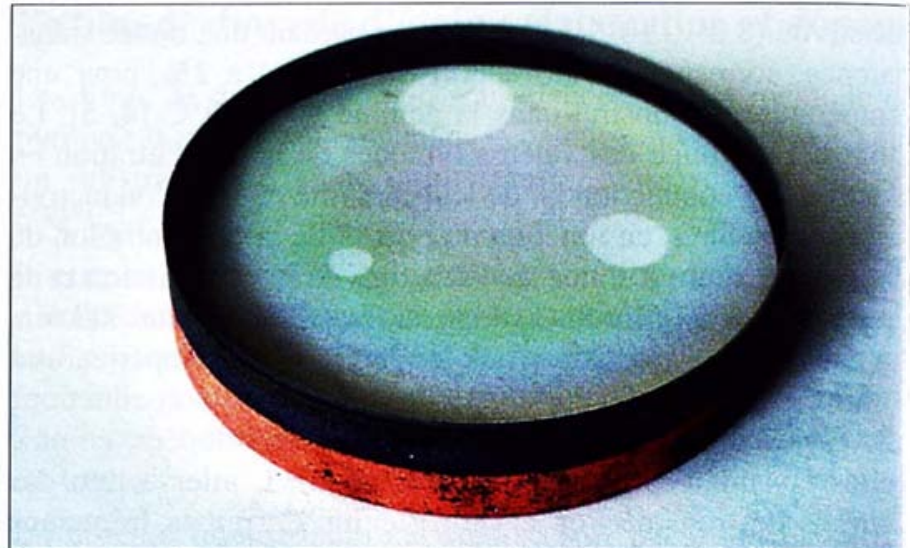

Fig. 6 - Vue d'un hublot recouvert d'un film bi-couche $\mathrm{SnO}_{2}: \mathrm{F}_{\text {et }} \mathrm{SnO}_{2}: \mathrm{Sh}$ conducteur et transparent, sauf dans les zones circulaires destinées à teste l'extension spatiale de la protection électrochimique. La piste latérale eı cuivre électrodéposé est également visible.

Fig. 6 - View of a lab-made window coated with a bi-layer $F$ and Sb-dope, $\mathrm{SnO}_{2}$ film with uncovered circular spots to check the spatial extension of th. electrochemical protection. The copper electrodeposited circular contact cai be seen close to the back face.

couche très conductrice $\mathrm{SnO}_{2}: \mathrm{F}$ à $570^{\circ} \mathrm{C}$ pendant 4 minutes partir d'une solution $0,2 \mathrm{M} \mathrm{SnCl}_{4}+0,14 \mathrm{M} \mathrm{NH}_{4} \mathrm{~F}$ dans l' méthanol. Cette première couche, de faible résistivité, a unı épaisseur d'environ $300 \mathrm{~nm}$. On dépose ensuite une couch، $\mathrm{SnO}_{2}: \mathrm{Sb}$ dopée à l'antimoine $(\mathrm{Sb} / \mathrm{Sn}=2 \%)$ à la même tempé rature de $570^{\circ} \mathrm{C}$ pendant 1 minute, d'une épaisseur de $100 \mathrm{~nm}$ à partir d'une solution $0,2 \mathrm{M} \mathrm{SnCl}_{4}+0,004 \mathrm{M} \mathrm{SbCl}_{3}$ dans lt méthanol. La résistance carrée du système bi-couche est di l'ordre de 10 ohms. Par la technique de spray, le dépôt a lieı non seulement en face avant mais aussi sur la paroi latérale dı hublot. Ceci permet d'établir un contact électrique latéral er cuivre, électrodéposé, soudable.

La figure 6 montre un exemple de réalisation d'un tel hublo muni de sa piste en cuivre circulaire près de la face arrière. Su la face avant, on peut noter la présence de zones circulaires dt différents diamètres $(3,6$ et $9 \mathrm{~mm})$ dans lesquelles la coucht d'oxyde a été enlevée par attaque chimique. Cette disposition : permis de vérifier l'étendue spatiale de la protection apportéc par la chloruration électrochimique. Les essais ont montri qu'elle était effective sur quelques millimètres mais pas au. delà. Il en résulte deux conséquences. D'une part, on peut main tenir propre une zone de quelques millimètres de diamètre pous le passage d'un faisceau de lumière par exemple. D'autre part l'effet de la chloruration n'est pas sensible au-delà de cette distance, respectant ainsi l'environnement. La figure 7 illustre de façon frappante l'efficacité de la protection électrochimique, er comparant l'état de deux hublots, l'un protégé, l'autre non après une immersion de 5 mois en rade de Brest. La figure $\varepsilon$ montre un bâti de test longue durée d'un hublot disposé devan une caméra réalisé par IFREMER. Il s'agit d'un essai mené sous contrôle potentiostatique, l'électrode étant polarisée ̀̀ $+1,5 \mathrm{~V} / \mathrm{ECS}$, ce qui correspond à une faible densité de courant de l'ordre de $10 \mu \mathrm{A} . \mathrm{cm}^{-2}$. Dans ces conditions, le procédé électrochimique est économe en énergie. Il peut l'être encore plus. dans la mesure où des essais, en cours d'expérimentation actuellement, tendent à indiquer qu'il ne serait pas nécessaire de maintenir en permanence la polarisation.

La polarisation en mode potentiostatique nécessite l'emploi d'électrodes auxiliaires, une contre-électrode, par exemple une grille en acier inoxydable, et une électrode de référence. I] 


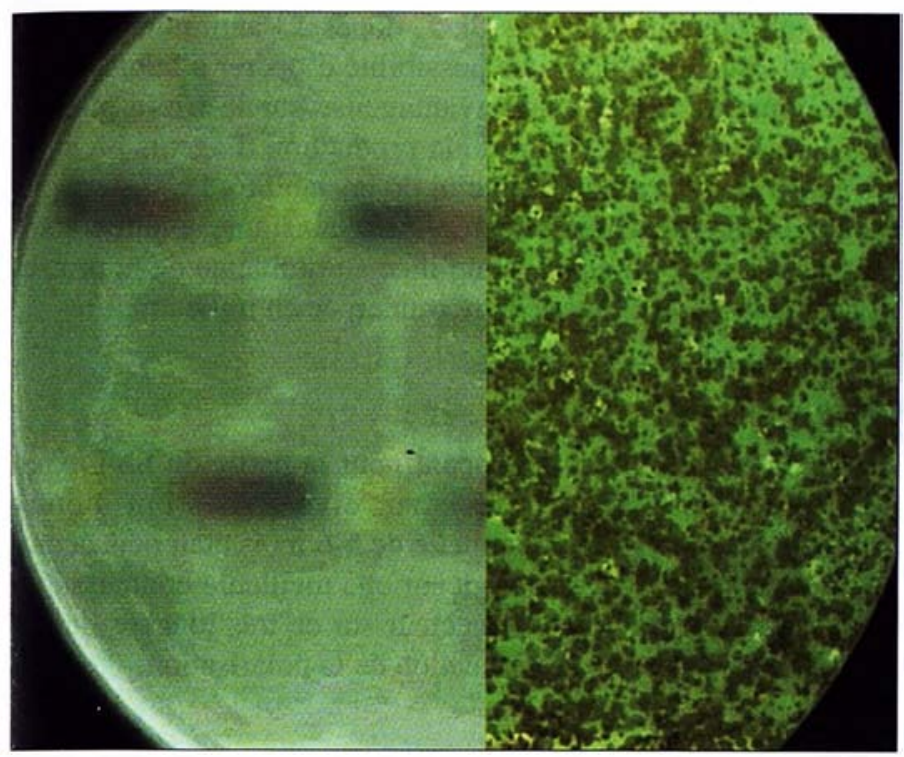

Fig. 7 - Effet d'une immersion de 5 mois par 10 mètres de fond en rade de Brest sur un hublot non protégé (à droite) et protégé (à gauche).

Fig. 7 - Macro views of reference (right) and $\mathrm{SnO}_{2}$ coated (left) windows after 5 months exposure.

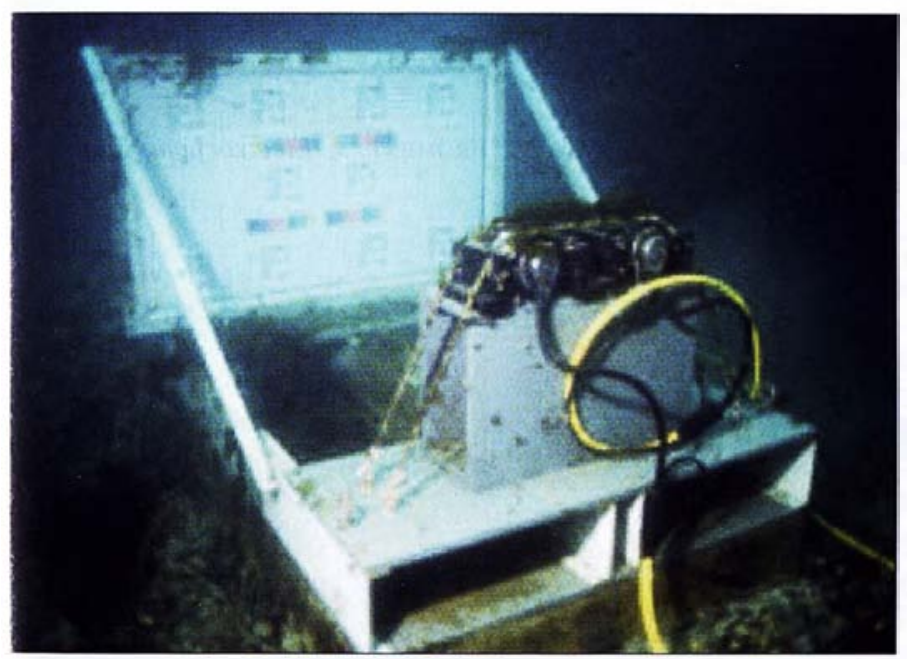

Fig. 8 - Vue d'un bâti immergé par 10 mètres de fond muni d'une caméra pour suivre l'état de la surface d'un hublot.

Fig. 8 - In situ window test experiment equiped with an on line video camera at the Marine Test Station of Ifremer Brest Centre.

s'agit là de contraintes tout à fait acceptables en laboratoire, beaucoup moins pour des essais sur site. C'est pourquoi on a développé un prototype de hublot comportant en face avant les trois électrodes requises. Le modèle réalisé est destiné à être placé devant une caméra de surveillance à longue focale. Il est présenté dans la figure 9. Le dispositif comprend une électrode de référence $\mathrm{Ag} / \mathrm{AgCl}$ et deux électrodes $\mathrm{SnO}_{2}: \mathrm{Sb}$ à $\mathrm{Sb} / \mathrm{Sn}=$ $2 \%$, de même surface, ce qui permet d'inverser le fonctionnement en cathode et en anode. La séparation des différentes zones a été réalisée par masquage et attaque chimique par le système $\mathrm{HCl}$ /poudre de zinc. Ce hublot a fait l'objet d'un premier test électrochimique en laboratoire. Polarisé à $+1,4 \mathrm{~V} / \mathrm{ECS}$ par rapport à l'électrode de référence $\mathrm{Ag} / \mathrm{AgCl}$, le courant circulant entre les deux électrodes $\mathrm{SnO}_{2}$ s'est avéré stable sur une période d'une vingtaine d'heures. Le fonctionnement de ce hublot intégrant les trois électrodes pose le problème de la stabilité du dioxyde d'étain en régime cathodique. L'oxyde peut en effet se détruire par réduction électrochimique suivie d'une

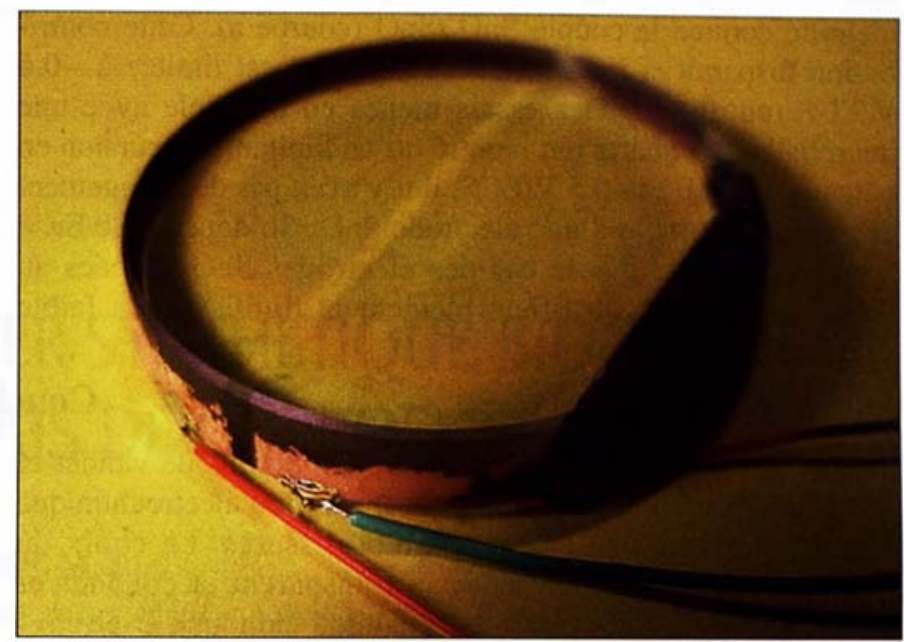

Fig. 9 - Prototype de hublot comportant les trois électrodes requises pour un fonctionnement en mode potentiostatique. Il comporte deux électrodes $\mathrm{SnO}_{2}$ de même surface et une électrode de référence de type $\mathrm{Ag} / \mathrm{AgCl}$. $\mathrm{J}$ a piste latérale en cuivre est segmentée pour alimenter les différentes électrodes.

Fig. 9 - View of a three-electrode window with the copper contact on the side, with two symetrical $\mathrm{SnO}_{2}$ electrodes and a $\mathrm{Ag} / \mathrm{AgCl}$ reference electrode.

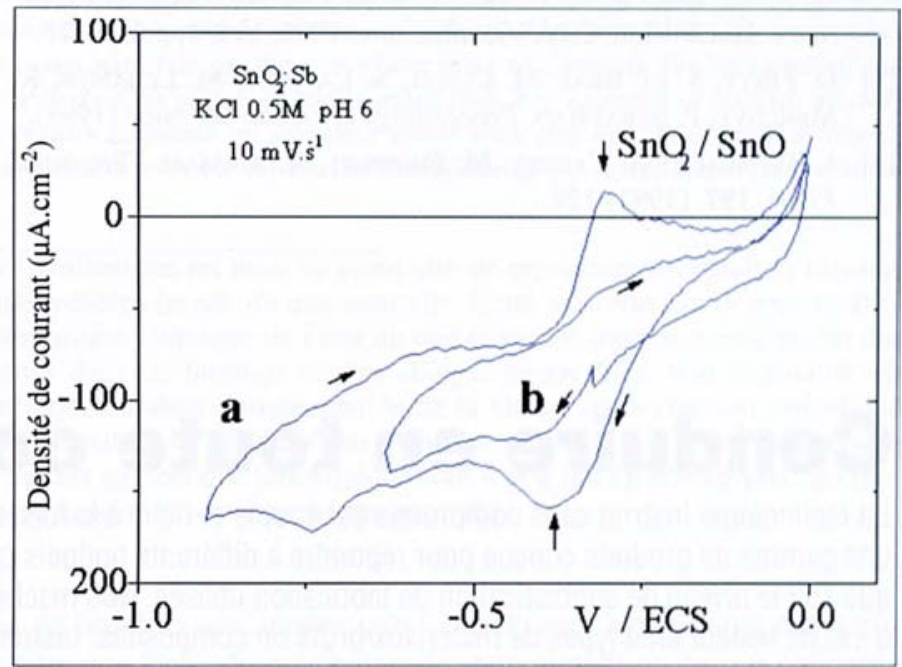

Fig. 10 - Voltampérogramme d'une électrode $\mathrm{SnO}_{2}$ dopée à l'antimoine $(\mathrm{Sb} / \mathrm{Sn}=2 \%$ ) polarisée entre 0 et $-0,9 \mathrm{~V} / \mathrm{ECS}$ (courbe a) et entre 0 et $-0,6 \mathrm{~V} / \mathrm{ECS}$ (courbe b) dans une solution $0,5 \mathrm{M} \mathrm{KCl}$ à $\mathrm{pH}$ 6. Vitesse de balayage: $10 \mathrm{mV} \cdot \mathrm{s}^{-1}$.

Fig. 10 - Current-voltage curves of a Sb-doped ( $\mathrm{Sb} / \mathrm{Sn}=2 \%) . \mathrm{SnO}_{2}$ electrode recorded at $10 \mathrm{mV} . \mathrm{s}^{-1}$ potential scan rate when the negative potential limit is: (a) -0.9 and (b) $-0.6 \mathrm{~V} / \mathrm{SCE}$.

oxydation lorsque l'on inverse les fonctions d'anode et de cathode. La réduction conduit à la formation de $\mathrm{SnO}$ et/ou d'étain métallique, espèces qui donnent lieu à des composés solubles par réoxydation. Cette difficulté peut heureusement être contournée en pratique, car la protection électrochimique ne nécessite qu'une faible densité de courant, $\approx 10 \mu \mathrm{A} . \mathrm{cm}^{-2}$. Ce faible courant provient de la réduction de l'oxygène dissous, plus particulièrement de la première étape de cette réaction qui conduit à la production du peroxyde d'hydrogène $\left(\mathrm{H}_{2} \mathrm{O}_{2}\right)$ qui est un agent biocide.

Le comportement électrochimique de $\mathrm{SnO}_{2}$ dans le domaine cathodique en solution $0,5 \mathrm{M} \mathrm{KCl}$ est illustré par la figure 10 . La réponse voltampérométrique dépend de la valeur de la borne négative du potentiel appliqué. Pour des potentiels allant jusqu'à $-0,9 \mathrm{~V} / \mathrm{ECS}$, on observe la réponse d'un couple redox 
identifié comme le couple $\mathrm{SnO}_{2} / \mathrm{SnO}$ (courbe a). Cette contribution disparaît si l'excursion de potentiel est limitée à $-0,6$ $\mathrm{V} / \mathrm{ECS}$ (courbe b). Des essais menés en parallèle avec une microbalance à quartz ont montré qu'en limitant l'excursion en potentiel entre 0 et $-0,5 \mathrm{~V} / \mathrm{ECS}$, il n'y avait pas de changement de masse mesurable d'une électrode $\mathrm{SnO}_{2}: \mathrm{Sb}$ dopée à $\mathrm{Sb} / \mathrm{Sn}=$ $2 \%$, ce qui n'est pas le cas des électrodes $\mathrm{SnO}_{2}$ dopées au fluor. Ces résultats justifient pleinement l'utilisation à faible densité de courant des films $\mathrm{SnO}_{2}$ dopés à l'antimoine comme anode et comme cathode. La possibilité d'opérer à faible densité de courant s'avère donc avantageuse sur le triple plan de l'économie d'énergie, celui de la production d'agents biocides à l'anode et à la cathode, et celui de la stabilité. Le système de hublot à trois électrodes intégrées offre ainsi une solution autonome et de mise en œuvre simplifiée au problème de la protection antisalissures d'une fenêtre immergée en milieu marin.

\section{Conclusions}

Les travaux présentés dans cet article ont permis de valider et de mettre en pratique le concept de protection électrochimique d'un hublot en verre contre les biosalissures. Le choix du dioxyde d'étain comme matériau transparent et conducteur s'est imposé pour des raisons de stabilité chimique et électrochimique. Les études en laboratoire ont fait ressortir l'intérêt de retenir l'antimoine comme dopant pour la partie du film expo sée à l'eau de mer. Les essais sur divers sites ont montr qu'une durée de vie opérationnelle de 5-6 mois était déjà acqui se; Les efforts à venir porteront sur une meilleure connaissanct du comportement du film protecteur sur de très longues durée: et suivant le protocole d'application de la polarisation.

\section{RÉFÉRENCES}

[1] M. Pourbaix, Atlas d'équilibres électrochimiques, Ed. GauthierVillars, Paris.

[2] T. El Moustafid, H. Cachet, B. Tribollet, D. Festy, N. LacotTE, Proceedings of the $11^{\text {th }}$ Asian-Pacific Corrosion Control Conference, HoChiMinh City, Vietnam, nov. 1999, Vol. 1, p.223-227.

[3] D. Festy, S. Le Bras, M. ClegG, N. Lacotte, M. Lehaitre, R. Menlove, P. Sebastiao, Proceedings of Ocean'98, Nice (1998).

[4] J. Bruneaux, H. Cachet, M. Froment, A. Messad, Thin Solid Films, 197, (1991) 129.
[5] A. Messad, H. Cachet, M. Froment, J. Bruneaux, J. Matel Sci., 29, (1994) 5095.

[6] Standard Potentials in Aqueous Solutions, IUPAC, éditeurs A.J Bard, R. Parsons et J. Jordan, Marcel Dekker Inc., New Yor; (1985).

[7] H. Cachet, M. Froment, F. Zenia, J. Electrochem. Soc., 14. (1996) 442.

[8] H. Cachet, F. Zenia, M. Froment, J. Electrochem. Soc., 14i (1999) 977.

\section{Conduire en toute confiance}

La technologie Instron sans compromis est souple et riche à la fois, avec une gamme de produits conçue pour répondre à différents budgets quel que soit le niveau de sophistication de fabrication utilisée. Nos machines d'essais testent tous types de matériaux bruts ou composites. Instron propose des machines d'essais de traction, torsion, compression, des machines d'essais de fatigue et de chocs ainsi que des systèmes d'essais de structures. Nos logiciels sont développés pour des applications simples ou encore de conception avancée. Prenez la bonne décision et standardisez sur la qualité, la fiabilité et le service après-vente Instron. Nous incarnons le meilleur choix pour des applications cruciales dans toutes les industries.

La différence Instron est dès à présent à votre portée. Visitez www.instron.com. The difference is measurable

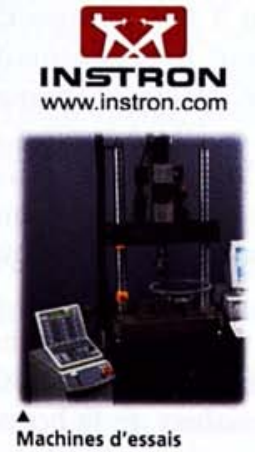

Servohydrauliques 8800

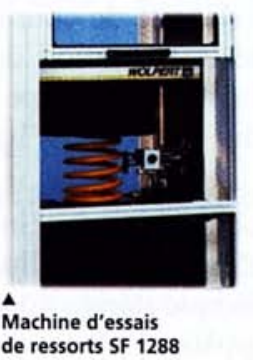
de ressorts SF 1288

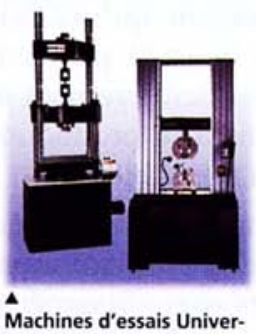

selles electromécaniques

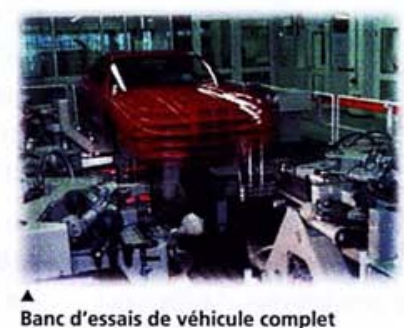

Banc d'essais de véhicule complet

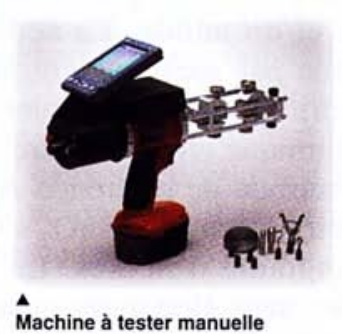

Machine à tester manuelle

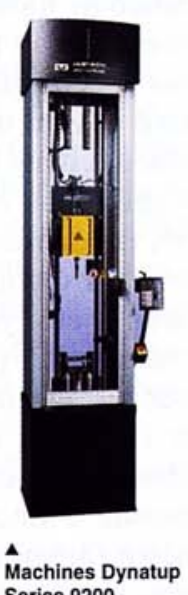

Series 9200

Instron SA - 11 Parc Club Ariane - 78284 Guyancourt Cedex - France - Tél: +01393066 30 - Fax: +013064 6711 - Email: info_query@instron.com 